\title{
Management interventions in dairy herds: Exploring within herd uncertainty using an integrated Bayesian model
}

\author{
Martin J. Green ${ }^{1,2 *}$, Graham F. Medley ${ }^{3}$, Andrew J. Bradley ${ }^{1}$, \\ William J. BROWNE ${ }^{4}$ \\ ${ }^{1}$ School of Veterinary Medicine and Science, University of Nottingham, Sutton Bonington Campus, \\ Sutton Bonington, LE12 5RD, United Kingdom \\ ${ }^{2}$ School of Mathematical Sciences, University of Nottingham, Nottingham, NG7 2RD, United Kingdom \\ ${ }^{3}$ Department of Biological Sciences, University of Warwick, Coventry, CV4 7AL, United Kingdom \\ ${ }^{4}$ Department of Clinical Veterinary Science, University of Bristol, Langford House, Langford, \\ Bristol, BS40 5DT, United Kingdom
}

(Received 19 August 2009; accepted 23 November 2009)

\begin{abstract}
Knowledge of the efficacy of an intervention for disease control on an individual farm is essential to make good decisions on preventive healthcare, but the uncertainty in outcome associated with undertaking a specific control strategy has rarely been considered in veterinary medicine. The purpose of this research was to explore the uncertainty in change in disease incidence and financial benefit that could occur on different farms, when two effective farm management interventions are undertaken. Bovine mastitis was used as an example disease and the research was conducted using data from an intervention study as prior information within an integrated Bayesian simulation model. Predictions were made of the reduction in clinical mastitis within 30 days of calving on 52 farms, attributable to the application of two herd interventions previously reported as effective; rotation of dry cow pasture and differential dry cow therapy. Results indicated that there were important degrees of uncertainty in the predicted reduction in clinical mastitis for individual farms when either intervention was undertaken; the magnitude of the $95 \%$ credible intervals for reduced clinical mastitis incidence were substantial and of clinical relevance. The large uncertainty associated with the predicted reduction in clinical mastitis attributable to the interventions resulted in important variability in possible financial outcomes for each farm. The uncertainty in outcome associated with farm control measures illustrates the difficulty facing a veterinary clinician when making an on-farm decision and highlights the importance of iterative herd health procedures (continual evaluation, reassessment and adjusted interventions) to optimise health in an individual herd.
\end{abstract}

disease control / dairy cow / uncertainty within herd / Bayesian model

\section{INTRODUCTION}

The decision to implement a particular control measure on a farm is influenced by a variety of factors including the expected

\footnotetext{
* Corresponding author: martin.green@nottingham.ac.uk
}

improvements in health, the perceived cost benefit of the intervention and the ease with which an intervention can be implemented. It can be difficult, however, to assess the likely impact of an intervention on a specific farm because of uncertainty associated with the intervention efficacy itself in a specific situation. One reason

This is an Open Access article distributed under the terms of the Creative Commons Attribution-Noncommercial License (http://creativecommons.org/licenses/by-nc/3.0/), which permits unrestricted use, distribution, and reproduction in any noncommercial medium, provided the original work is properly cited. 
for this uncertainty is that farms are different, for example, in herd size, disease status, population structures (e.g. cow numbers, age and calving pattern), policies for disease management and general management factors (such as the approach to nutrition). Uncertainty about the clinical and financial benefit of an intervention, will affect the decision as to whether or not to implement the intervention on an individual farm. Therefore, in terms of making decisions on preventive measures, an understanding of the uncertainty in the likely outcome on an individual farm is essential, and yet rarely considered in veterinary medicine.

Mastitis remains an important disease of dairy cattle worldwide that results in financial losses, welfare infringements and potentially public health repercussions [2]. Philosophically, high levels of endemic disease in animals farmed for milk production is unacceptable to modern society, yet it is not clear that substantial progress has been made in reducing the incidence or prevalence of mastitis in recent years. As a result, some countries have recently commenced national research schemes in an attempt to improve the situation ${ }^{1,2,3}$. In this paper we take the example of dairy cow mastitis to examine the uncertainty in outcome associated with making specific interventions for disease control.

A variety of studies have examined and reported risk factors for bovine mastitis (e.g. [1, 11-14] although unfortunately few factors identified in observational studies have been subsequently tested in controlled intervention

\footnotetext{
${ }^{1}$ National udder health project of the Netherlands, [on line] (2009) http://www.gddeventer.com/ templates/dispatcher.asp?opage_id $=5225633 \&$ location $=913122341975058,1062573$, true\&page_id $=2$ 5250914 [consulted August 2009].

${ }^{2}$ Canadian bovine mastitis research network, [on line] (2008) http://www.medvet.umontreal.ca/ reseau_mammite/display.php?lang=en [consulted August 2009].

${ }^{3}$ UK DairyCo National Mastitis Control Plan, [on line] (2009) http://www.mastitiscontrolplan.co. uk/ index.php [consulted August 2009].
}

studies to provide direct evidence of causality. However, a recent randomised clinical trial in the UK reported that a systematic mastitis intervention plan reduced the incidence of clinical mastitis [9] and subsequent analysis identified dry period factors important in mastitis control [8].

The purpose of this research was to explore the uncertainty in the change in mastitis incidence and financial benefit that could occur on different farms, when two effective dry period management interventions are undertaken. The research was conducted using data from the earlier intervention study as prior information within an integrated Bayesian simulation model, to make predictions for individual farms, of the impact of undertaking these interventions.

\section{MATERIALS AND METHODS}

\subsection{The data}

Data to estimate prior distributions for use in the simulation model were taken from a recent randomised clinical trial that was carried out on 52 dairy farms in England and Wales. This original study comprised the application of a mastitis control plan on 26 of the farms and resulted in a mean reduction in the overall incidence rate of clinical mastitis of approximately $20 \%$ on the intervention farms compared to controls [9]. A subsequent analysis of these data revealed large between farm heterogeneity in the incidence rate of first cases of clinical mastitis in the first 30 days of lactation (FCM30), and dry period management measures were identified that had a significant impact on this rate [8]. The initial research produced estimates of mean effects of interventions on FCM30 across all farms.

In this current study, two particular mastitis preventive measures were considered in more detail to investigate their effect on predicted individual farm clinical mastitis incidence; the use of differential dry cow therapy and a method of pasture rotation for the dry cows. Differential dry cow therapy was a management strategy in which different groups of cows at drying off were subjected to different therapeutic regimes, according to cell count status at drying off. Cows with raised somatic cell counts (typically over 200000 cells per $\mathrm{mL}$ in any one of 
Table I. Summary of relevant parameters in the multilevel logistic regression model, with clinical mastitis in the first 30 days of lactation as the response for cows at pasture during the dry period [8].

\begin{tabular}{lcccc}
\hline Model terms & Variance & Odds ratio & \multicolumn{2}{c}{$\begin{array}{c}95 \% \text { Credibility } \\
\text { interval }\end{array}$} \\
\hline Intercept $=-0.84$ & & & $2.5 \%$ & $97.5 \%$ \\
Intervention covariates included & & & \\
Select one dry cow treatment for whole herd & Reference \\
Select different dry cow treatments for individual cows & & 0.74 & 0.55 & 0.97 \\
Pasture grazing policy other than "rest 4 graze 2"1 & & Reference & & \\
Pasture grazing policy is "rest 4 graze 2"1 & & 0.32 & 0.11 & 0.90 \\
Between farm-year & 0.09 & & 0.02 & 0.21 \\
Between farm & 0.22 & & 0.09 & 0.41 \\
Between (Farm * Parity 1) & 0.43 & & 0.40 & 0.82
\end{tabular}

Other significant cow level covariates included in the model were: parity of cow, and the presence of raised somatic cell counts within 90 days before the time of drying off.

Other significant farm covariates included in the model were: whether a California Milk test was used to test milk of cows after calving, what type of fly control measures were applied and whether or not vaccination of the herd was undertaken with a Leptospirosis Vaccine.

1 "Rest 4 graze 2" - a pasture rotation method such that dry cows graze the pasture for a maximum of 2 weeks followed by stock not grazing the pasture for a minimum of 4 weeks.

the last three recordings of lactation) were treated differently to other cows, the different treatments being directed towards specific pathogens, according to individual farm history [3]. In the original research, cows on farms that implemented this policy had a reduced odds of a case of clinical mastitis in the first 30 days of lactation, for dry cows at pasture, with a mean of odds ratio of 0.74 (95\% credible inter$\mathrm{val}=0.55-0.97$, indicating a probability of 0.95 that the true value was within this range). Similarly, in the original research, cows that were on farms that had a specific grazing policy for dry cows were at reduced risk of clinical mastitis. Cows on farms in which the pasture was grazed for a maximum of 2 weeks, followed by a rest period from grazing of 4 weeks, were at reduced odds of a case of clinical mastitis in the first 30 days of the next lactation, compared to herds that did not implement this policy, with a mean odds ratio of $0.32(95 \%$ credible interval $=0.11-0.90)$. The original model, as described by Green et al. [9] with relevant parameter estimates is summarised in Table I.

The question for the current research was that, conditional on the estimated impact on clinical mastitis of these interventions, on other farm characteristics and on the estimated cost of clinical mastitis, what was the probability distribution (degree of uncertainty) of the outcome (reduction in number and cost of clinical mastitis cases) for each individual farm that implements the interventions?

\subsection{Analysis}

The analysis contained two main elements integrated into a single, unified Bayesian model, based on the method described by Spiegelhalter et al. [16]. The first element was a replication of the analysis of the original study data to derive the full probability distributions of necessary parameters. The second element comprised propagating these parameter distributions through a financial model, to investigate the costs attributable to changes in mastitis incidence, for each farm. This method was used so that the full joint probability distributions of all parameters were taken into account [16] and the modelling process is described in detail below. A diagrammatic overview of the analysis is provided in Figure 1.

The original research that we have used to derive prior distributions has been described previously [8] and consisted of a multilevel logistic regression model [7], specified so that correlations within the data (cow-dry periods within years within farms) were accounted for appropriately. Cows were categorised as 1 (a case of clinical mastitis within 30 days of 


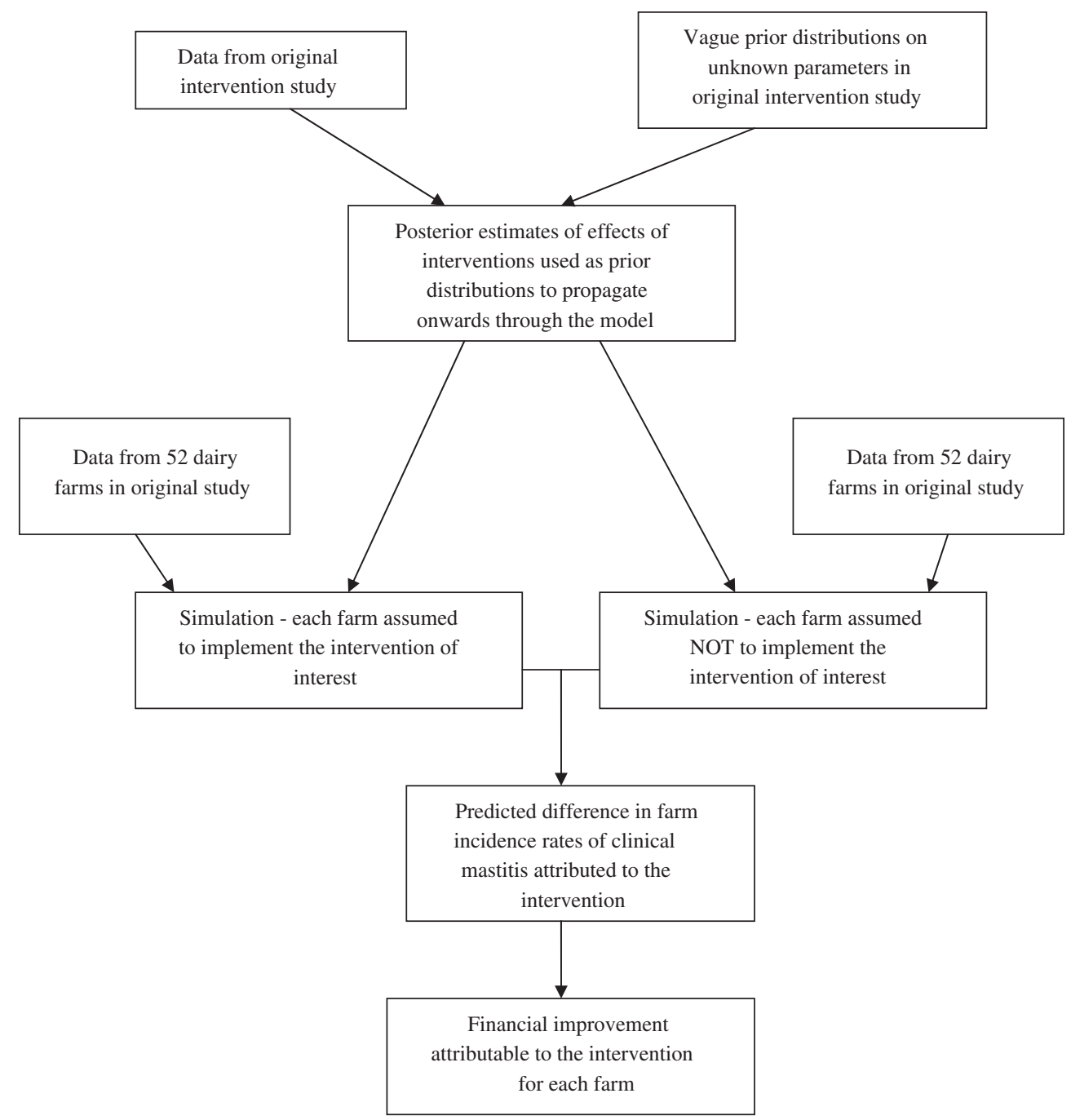

Figure 1. Overview of the integrated Bayesian model used to make predictions of the change in individual farm incidence of clinical mastitis.

calving) or 0 (no case of clinical mastitis within 30 days of calving). During the 2 years of data collection, year 1 comprised information gathered prior to any interventions being carried out and year 2 comprised information collected following the implementation of the mastitis control plan on 26 herds, selected at random. The logistic model specification can be summarised as:

First case of clinical mastitis (CM) within 30 days of calving (FCM30):

$$
\begin{aligned}
& \operatorname{FCM} 30_{i j k}(1=\mathrm{CM}, 0=\text { no CM }) \\
& \begin{aligned}
& \sim \text { Bernoulli probability }\left(\text { mean }=\pi_{i j k}\right) \\
& \operatorname{Logit}\left(\pi_{i j k}\right)= \alpha+\beta_{1} \mathrm{X}_{i j k}+\beta_{2} \mathrm{X}_{j k}+\beta_{3} \mathrm{X}_{k} \\
&+u_{j k}+v_{k}
\end{aligned}
\end{aligned}
$$$$
u_{j k} \sim \text { normal distribution }\left(0, \sigma_{\mathrm{u}}^{2}\right)
$$$$
v_{k} \sim \operatorname{normal} \text { distribution }\left(0, \sigma_{\mathrm{v}}^{2}\right)
$$ 
where the subscripts $i, j$ and $k$ denote the $i^{\text {th }}$ cowdry period, the $j^{\text {th }}$ farm-year of study and the $k^{\text {th }}$ farm, respectively, $\pi_{i j k}$ the fitted probability of FCM30 for cow-dry period $i$ of year $j$ on farm $k$, $\alpha$ the regression intercept, $X_{i j k}$ the vector of covariates at cow-dry period level, $\beta_{1}$ the coefficients for covariates $\mathrm{X}_{i j k}, \mathrm{X}_{j k}$ the vector of year level covariates, $\beta_{2}$ the coefficients for covariates $\mathrm{X}_{j k}, \mathrm{X}_{k}$ the vector of farm-level covariates, $\beta_{3}$ the coefficients for covariates $\mathrm{X}_{k}, v_{k}$ the random effect to reflect residual variation between farms and $u_{j k}$ the random effect to reflect residual variation between years. Covariate selection and adequacy of model fit using posterior predictions, have been described in detail previously [8].

The next component of the analysis comprised inclusion of the parameter distributions from the above model (Eq. 1) and known farm data (such as the cow parity, somatic cell count information and preventive measures undertaken), within an integrated Markov chain Monte Carlo (MCMC) procedure, to simulate the outcome in FCM30 for each of the farms in year 2. Each cow (and from this each farm) had two predictions made in the simulation; one using the assumption that the intervention was carried out on the farm, and one using the assumption that the intervention was not carried out. An estimate of the effect of each intervention on each farm was then made by calculating the difference between the predicted occurrence of clinical mastitis in year 2 with or without the intervention being undertaken. The predicted difference in mastitis incidence for each farm was calculated at each iteration of the integrated MCMC procedure. The predicted incidence of clinical mastitis for individual cows and farms was estimated as follows:

$$
\begin{aligned}
& \text { PREDICTED FCM } 30_{i j k}(1=\mathrm{CM}, 0=\operatorname{not} \mathrm{CM}) \\
& \quad \sim \text { Bernoulli probability }\left(\mathrm{P}_{i j k}\right) \\
& \text { Logit }\left(\mathrm{p}_{i j k}\right)=\alpha+\beta_{1} \mathrm{X}_{i j k}+\beta_{2} \mathrm{X}_{j k}+\beta_{3} \mathrm{X}_{k}+v_{k}+u_{j k} .
\end{aligned}
$$

Where $\mathrm{P}_{i j k}$ is the predicted probability of FCM30 for cow-dry period $i$ of year $j$ on farm $k$ and PREDICTED FCM $30_{i j k}$ is a draw from the Bernoulli distribution with probability $\mathrm{P}_{i j k}$. All other parameters are described previously (Eq. 1). The farm data for the 2 interventions were used in 2 ways; the data were firstly used to indicate that an intervention was implemented on each farm and a prediction made for year 2 of the study. The data for the intervention were reversed for year 2 of the study, to provide a prediction for clinical mastitis under the assumption that the intervention on each farm had not been undertaken. After model convergence (minimum of 2000 iterations), at each iteration of the MCMC process, the values of PREDICTED FCM30 ${ }_{i j k}$ for the individual cows were summed across each farm in year 2 , and the predicted incidence of clinical mastitis with the intervention undertaken was subtracted from the predicted incidence without the intervention undertaken to provide an estimate for each farm, of the difference in clinical mastitis attributable to each intervention. After model convergence, 8000 iterations were used to make predictions from the model; this number of iterations was deemed to be clearly sufficient to adequately summarise model output parameter distributions.

An estimated cost of a case of clinical mastitis was incorporated into the model and based on a recent publication of disease costs in UK dairy herds [6]. The mean estimated cost per case was a combination of treatment costs (including veterinary time), herdsman time, discarded milk, reduced subsequent milk yield, severity of disease and risk of culling or death. Milk price has recently changed in the UK, however, and there is some variation between farms. Therefore a distribution for milk price was included in the calculation, based on current prices, with a mean of $£ 0.25$ per litre and standard deviation of $£ 0.01$ per litre. The resultant cost of a case of clinical mastitis was normally distributed with mean $£ 212.30$ and standard deviation $£ 5.44$. The predicted difference in the financial cost of clinical mastitis for each farm attributable to the change in mastitis incidence between intervention and non-intervention policies was estimated as:

Change in cost for Farm $\mathrm{k}$ in year $2=\left(\mathrm{FCM}_{3} \mathrm{O}_{2 k}\right.$ int-FCM $30_{2 k}$ noint)* cost per case.

Where "FCM $30_{2 k}$ int" was the total number of cases of FCM30 in year 2 for Farm $k$ assuming the intervention was applied and "FCM $30_{2 k}$ noint" was the total number of cases of FCM30 in year 2 for Farm $_{k}$ assuming the intervention was not applied, and cost per case is the estimated cost of a case of FCM30. Therefore a distribution for the change in cost attributable to each intervention for each farm was obtained from the integrated model.

\section{RESULTS}

\subsection{Predicted impact of pasture rotation on the incidence of clinical mastitis}

To illustrate the predicted change in FCM30 on each farm attributable to pasture rotation, posterior distributions of this predicted change 


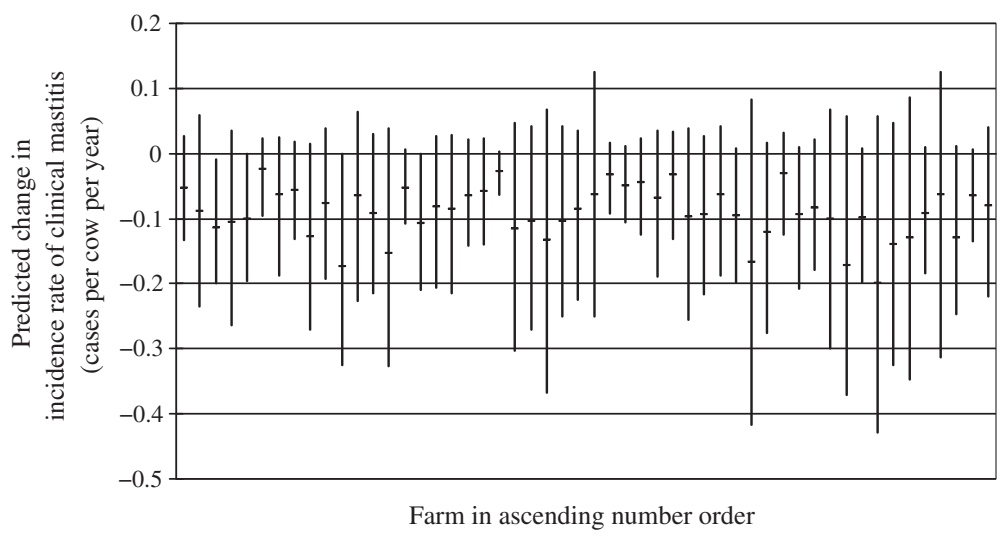

Figure 2. Median and $95 \%$ credibility intervals for the predicted change in incidence rate of clinical mastitis cases in the first 30 days of lactation on each farm, attributable to the intervention of pasture rotation, estimated from the integrated Bayesian model.

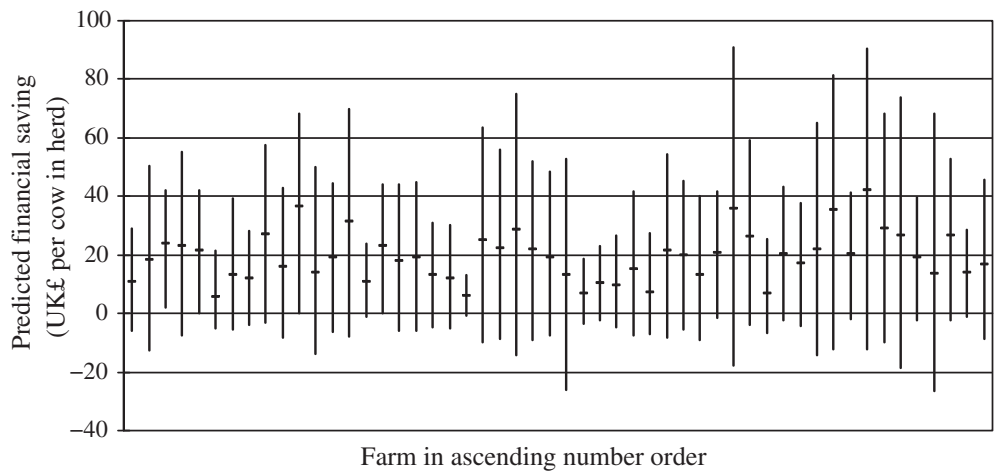

Figure 3. Median and $95 \%$ credibility intervals for the predicted financial saving ( $£$ per cow per year) for each farm, attributable to the intervention of pasture rotation, estimated from the integrated Bayesian model.

are presented in Figure 2. The mean reduction in median incidence rate over all farms was 0.09 cases per cow per year. There was notable uncertainty in the estimated reduction in FCM30 for all farms and the $95 \%$ credible intervals ranged in size from 0.07 to 0.50 cases per cow per year. The mean size of the $95 \%$ credibility intervals for the FCM30 across all farms was 0.26 cases per cow per year.

The total predicted financial saving per cow in the herd per year, from implementation of the pasture rotation policy, is presented in
Figure 3. There was substantial within farm uncertainty in predicted financial saving per cow and the mean size of the $95 \%$ credibility intervals for annual saving across all farms was $£ 54.24$ per cow in the herd. The $95 \%$ credibility intervals for individual farms ranged in size from $£ 13.86$ to $£ 108.50$. The uncertainty in individual farm total financial saving was further explored by assessing the probability, for each farm as a whole, that the total saving would exceed $£ 500$ or $£ 1000$ per year. For the majority (46/52) farms there was 

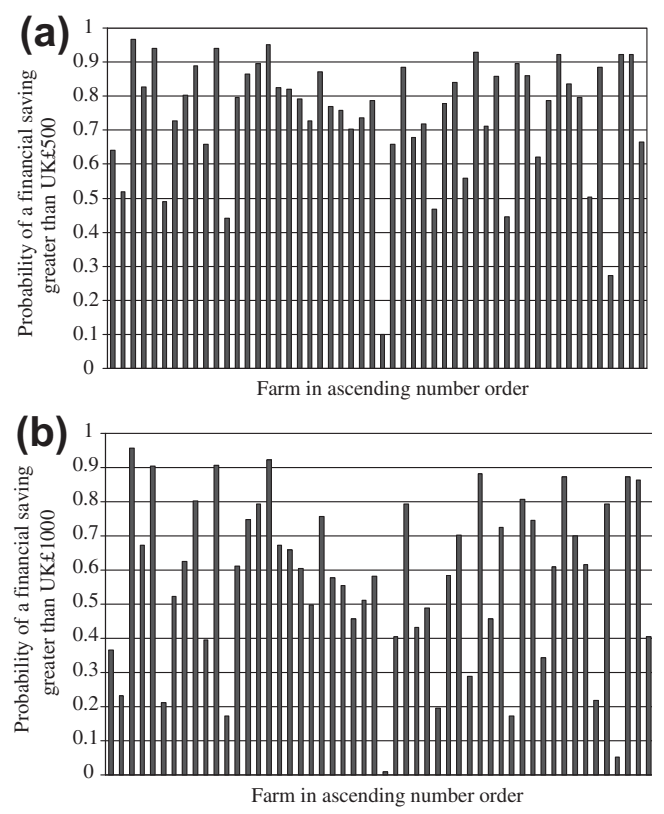

Figure 4. Bar charts illustrating the probability, estimated from the integrated model, that a financial saving on each farm per annum, attributable to implementation of pasture rotation, would exceed $£ 500$ (a), or $£ 1000$ (b).

a probability $>0.50$ of a saving of at least $£ 500$ per year and for 33 farms the probability of a return in excess of $£ 1000$ was $>0.50$ (Fig. 4).

\subsection{Predicted impact of approach to dry cow therapy on the incidence of clinical mastitis}

Posterior estimates of the difference in the FCM30 attributable to differential dry cow therapy for each of the 52 farms, is presented in Figure 5. The mean reduction in median incidence rate across all farms was 0.03 cases per cow per year. There was notable uncertainty in the estimated reduction in FCM30 for all farms and the $95 \%$ credible intervals ranged in size from 0.08 to 0.54 cases per cow per year. The mean size of the $95 \%$ credibility intervals for the FCM30 across all farms was 0.26 cases per cow per year.

The total predicted financial saving per cow in the herd per year, from implementation of differential dry cow therapy policy for each farm, is presented in Figure 6. There was substantial within farm uncertainty in predicted financial saving per cow and the mean size of the $95 \%$ credibility intervals for annual saving across all farms was $£ 55.47$ per cow. The $95 \%$ credibility intervals for each farm ranged in size from $£ 16.62$ to $£ 111.83$. The total financial saving was further explored by assessing the probability, for each farm, that the total saving would exceed $£ 500$ or $£ 1000$ per year. For 20 out of the 52 , farms there was a probability $>0.50$ of a saving of at least $£ 500$ per year but for only 6 farms was the probability of a return in excess of $£ 1000>0.50$ (Fig. 7).

\section{DISCUSSION}

There was important within farm uncertainty associated with the predicted change in FCM30 and anticipated financial gains from implementing the interventions examined in this research. This reflects uncertainty of the intervention efficacy in different farm situations (such as herd differences in the parity of cows, the cow somatic cell counts prior to drying off and other aspects of herd management). The posterior estimates from the Bayesian model incorporate the full joint probability distributions of all model parameters and the estimate of the impact of the interventions is conditional on these parameter distributions for each farm. A greater size and certainty in the efficacy of an intervention would result in a greater certainty in the anticipated financial gains, and thus the original strength of evidence for an intervention is an important component of the model. The true uncertainty associated with each intervention is slightly larger than estimated in the models presented, because of unattributed uncertainty in the cost of mastitis. Accurate data for mastitis costs (such as the quantity of discarded milk and reduced subsequent milk yield) were not available and thus it was decided could not be safely included in the models.

The uncertainty in outcome on a farm when implementing interventions demonstrates the difficulty for a clinician when attempting to establish cost effective herd health schemes on 


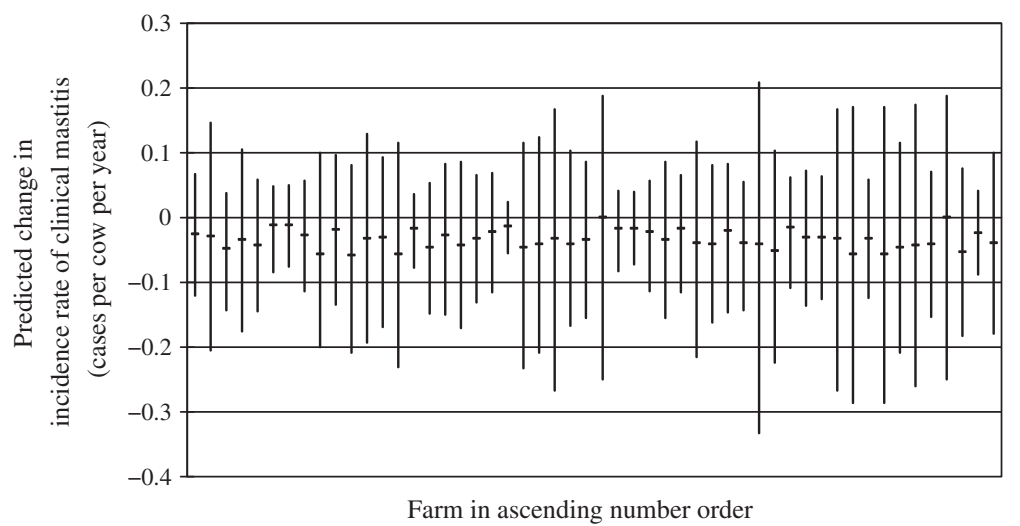

Figure 5. Median and $95 \%$ credibility intervals for the predicted change in incidence rate of clinical mastitis cases in the first 30 days of lactation on each farm, attributable to the intervention of differential dry cow therapy, estimated from the integrated Bayesian model.

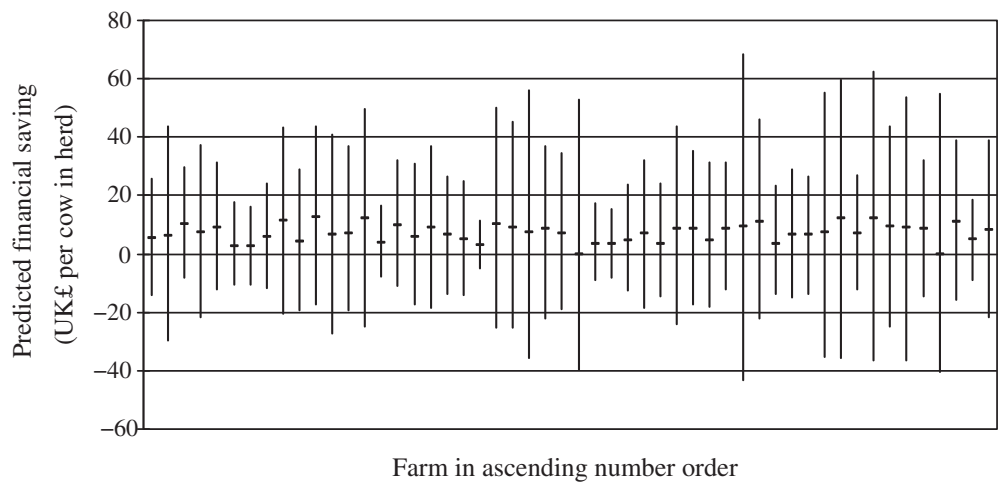

Figure 6. Median and $95 \%$ credibility intervals for the predicted financial saving ( $£$ per cow per year) for each farm, attributable to the intervention of differential dry cow therapy, estimated from the integrated Bayesian model.

an individual unit. A practical consequence of such uncertainty means that an iterative herd health procedure is essential [4], that is continual evaluation, reassessment and further interventions may be required to identify the best route for management change in a specific herd. An improved understanding of when and how interventions are best applied on individual farms would be of great clinical benefit and an increased number of intervention studies of farm animal health would help to achieve this. The decision to implement an intervention on a specific farm is necessarily complex with differences in success likely between farms and uncertainty of success within farm. A further complication, as illustrated in this example, is whether an intervention is applied to individual cows (such as dry cow therapy), or is a farm policy (pasture rotation) and this may also affect a decision on whether to implement a particular management intervention. Unless interventions are relatively cheap, easy to implement and considered to have a reliably large return on investment, these complexities may make the 

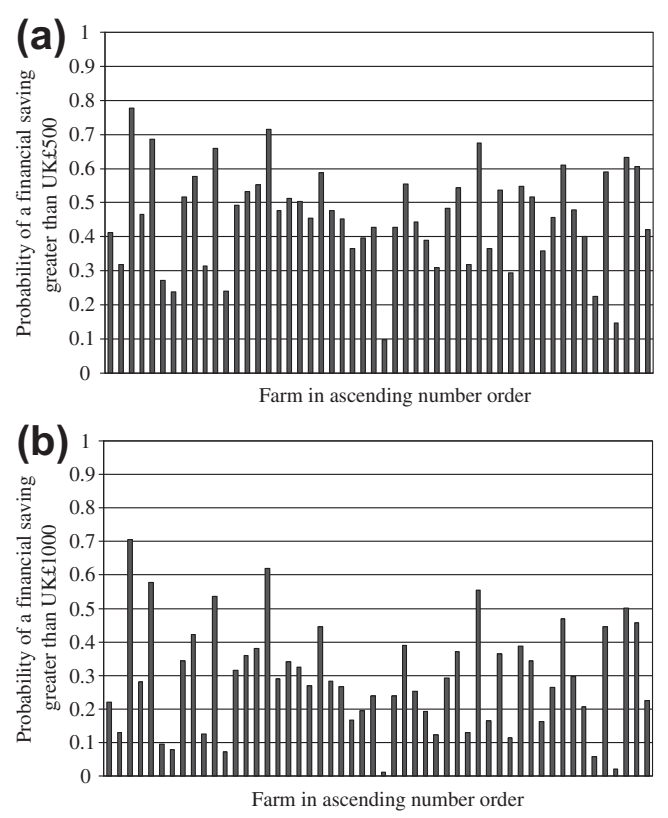

Figure 7. Bar charts illustrating the probability, estimated from the integrated model, that a financial saving on each farm per annum, attributable to implementation of differential dry cow therapy, would exceed $£ 500$ (a), or $£ 1000$ (b).

decision process complex and result in a cautious approach.

The uncertainty in clinical and financial outcome for an individual farm is important and illustrates the usefulness of linking a probability density (risk) to anticipated financial returns. The probability of different financial returns from implementing the interventions (Figs. 4 and 7), show large differences between farms in the probability of returns of $£ 500$ or $£ 1000$. These financial returns can be considered as a "room for investment" to implement the control measure and suggest that few farms can be very certain (probability $>0.80$ ) of a financial gain $\geq £ 1000$ per annum for either intervention. A decision to carry out an intervention will depend on a combination of the magnitude of expected financial return and the risk acceptance of the decision maker. The farm specific costs to implement the two interventions, pasture rotation and differential dry cow therapy were unknown in this study. Rotating dry cow pasture is a farm-level intervention and would involve aspects of grazing management, such as moving fences, feeders and moving cow groups on a fortnightly basis. By contrast, implementing differential dry cow therapy is a cow level intervention and involves time to analyse each individual cow health record at drying off and to select the appropriate therapeutic regime. In this example, neither of these costs are likely to be large, probably less than $£ 500$ per annum, but such costs should be identified and used to offset expected benefits as part of decision on whether to undertake an intervention.

Clinical decisions on disease prevention will contain further heterogeneity because of personal elements specific to the individuals involved, such as the decision makers current physiological, psychological, social and emotional state [10]. Different approaches towards financial outlay and risk aversion are likely and if captured, such probabilities could be added within the Bayesian framework [16].

The integrated Bayesian model used for this analysis incorporated the full joint probability distributions of all parameters and is thus different to a traditional two-step approach that initially formulates a risk model and separately uses the model parameters to predict future disease and financial outcomes [16]. The integrated model simultaneously derives the joint posterior distribution for all unknown parameters and propagates the effects through the predictive model. In this example, uncertainty in the prediction of mastitis for each herd is included as well as the uncertainty of the effects of the interventions. There are several advantages of this approach [16]. First, appropriate dependence between unknown probabilities is propagated automatically through the model rather than making assumptions about parameter independence or making assumptions about the dependence structure [5]. Second, there is no need to make distributional assumptions about the parameters that are propagated from an initial model (incorporating data and prior distributions) through into the predictive element. The main disadvantage of the unified Bayesian approach is the need for full MCMC software in order to obtain a solution although 
this is now freely available [17]. Another potential disadvantage is that it can be difficult to evaluate or check model accuracy. In this example, data from a large number of different farms would be required, both before and after the intervention, to evaluate the model using a cross validation-type approach. However, such data are often not available. Such unified Bayesian models have been used in human medicine [15] but rarely in veterinary medicine and they provide a useful method to improve the understanding of the uncertainties involved in clinical decision making.

Acknowledgements. This research was funded by the Wellcome Trust - Martin Green is a Wellcome Trust Intermediate Clinical Fellow. We would like to thank the Milk Development Council (now DairyCo, UK) for funding the initial research.

\section{REFERENCES}

[1] Barkema H.W., Schukken Y.H., Lam T.J., Beiboer M.L., Benedictus G., Brand A., Management practices associated with the incidence rate of clinical mastitis, J. Dairy Sci. (1999) 82:1643-1654.

[2] Bradley A.J., Bovine mastitis: an evolving disease, Vet. J. (2002) 164:116-128.

[3] Bradley A.J., Huxley J.N., Green M.J., A rational approach to dry cow therapy II-Making logical treatment decisions, In Pract. (2003) 25:12-17.

[4] Brand A., Noordhuizen J.P., Schukken Y.H., Herd health and production management in dairy practice, Wageningen Pers, Wageningen, The Netherlands, 1996.

[5] Chessa A.G., Dekkae R., van Vliet B., Steyerberg E.W., Habbema J.D.F., Correlations in uncertainty analysis for medical decision making: an application to heart valve replacement, Med. Decis. Making (1999) 19:276-286.

[6] Esslemont R.J., Kossaibati M.A., DAISY Research Report No. 5, University of Reading, UK, 2002.
[7] Goldstein H., Multilevel Statistical Models, Edward Arnold, London, 1995.

[8] Green M.J., Bradley A.J., Medley G.F., Browne W.J., Cow, farm and management factors during the dry period that determine the rate of clinical mastitis after calving, J. Dairy Sci (2007) 90:3764-3776.

[9] Green M.J., Leach K.A., Breen J.E., Green L.E., Bradley A.J., National intervention study of mastitis control in dairy herds in England and Wales, Vet. Rec. (2007) 160:287-293.

[10] Hastie R., Dawes R.A., Rational choice in an uncertain world - The psychology of judgement and decision making, Sage Publications Inc., California, USA, 2001.

[11] O'Reilly K.M., Green M.J., Peeler E.J., Fitzpatrick J.L., Green L.E., Investigation of risk factors for clinical mastitis in British dairy herds with bulk milk somatic cell counts less than 150,000 cells/ ml, Vet. Rec (2006) 158:649-653.

[12] Peeler E.J., Green M.J., Fitzpatrick J.L., Morgan K.L., Green L.E., Risk factors associated with clinical mastitis in low somatic cell count British dairy herds, J. Dairy Sci. (2000) 83:2464-2472.

[13] Schukken Y.H., Erb H.N., Sears P.M., Smith R.D., Ecologic study of the risk factors for environmental mastitis in cows, Am. J. Vet. Res. (1988) 49:766-769.

[14] Schukken Y.H., Grommers F.J., Van de Geer D., Erb H.N., Brand A., Risk factors for clinical mastitis in herds with a low bulk milk somatic cell count. 1. Data and risk factors for all cases, J. Dairy Sci. (1990) 73:3463-3471.

[15] Spiegelhalter D.J., Best N., Bayesian approaches to multiple sources of evidence and uncertainty in complex cost-effectiveness modelling, Stat. Med. (2003) 22:3687-7309.

[16] Spiegelhalter D.J., Abrams K.R., Myles J.P., Bayesian approaches to clinical trials and healthcare evaluation, Statistics in practice, John Wiley and Sons, Chichester, UK, 2004, pp. 305-347.

[17] Spiegelhalter D.J., Thomas A., Best N., WinBUGS Version 1.4.1, MRC Biostatistics Unit, Cambridge, UK, 2004. 